Revue d'histoire de l'Amérique française

ZRS REVUE D.HISTOIRE DE L'AMÉRIQUE FRANÇAISE

\title{
La troublante altérité de l'histoire Réflexion sur le passé comme « Autre » radical
}

Jean-Marie Fecteau

Volume 59, numéro 3, hiver 2006

URI : https://id.erudit.org/iderudit/013083ar

DOI : https://doi.org/10.7202/013083ar

Aller au sommaire du numéro

Éditeur(s)

Institut d'histoire de l'Amérique française

\section{ISSN}

0035-2357 (imprimé)

1492-1383 (numérique)

Découvrir la revue

\section{Citer cet article}

Fecteau, J.-M. (2006). La troublante altérité de l'histoire Réflexion sur le passé comme " Autre " radical. Revue d'histoire de l'Amérique française, 59(3),

333-345. https://doi.org/10.7202/013083ar d'utilisation que vous pouvez consulter en ligne.

https://apropos.erudit.org/fr/usagers/politique-dutilisation/ 


\section{La troublante altérité de l'histoire Réflexion sur le passé comme "Autre " radical}

JEAN-MARIE FECTEAU

Département d'histoire

Université du Québec à Montréal

$\mathbf{R}$ éfléchir sur le statut de l'«Autre» dans une perspective historienne une véritable fixation sur l'«identité», ce contraire de l'Autre, le concept d'altérité ne risque-t-il pas de perdre en crédibilité scientifique ce qu'il gagnerait en popularité? Mais il ne s'agit pas seulement d'évaluer la pertinence conceptuelle d'un terme utilisé de plus en plus à toutes les sauces. Il faut aussi prendre la mesure des rapports entre la pratique historienne et le concept d'altérité.

\section{L'EXCLU ET LE MARGINAL COMME «AUTRE »?}

Le réflexe le plus «naturel», pour un historien des systèmes de régulation sociale et de la marginalité, est en effet d'assigner à l'Autre, comme concept, le sens d'un rejet à la marge, d'une exclusion, un peu comme le faisait le Foucault première manière de l'Histoire de la Folie. Mais on peut tout aussi bien remettre en question la pertinence heuristique du concept d'altérité dans ce domaine.

L'Autre de l'exclusion sociale, effet des multiples césures issues des sociétés inégalitaires, est un Autre qui est produit. Il a très peu de choses à voir avec l'Autre d'un Lévinas (nous y reviendrons), et même si les populations ainsi qualifiées ont un certain rôle, ambigu, dans la construction des identités sociales, c'est moins en raison de leur étrangeté fonda- 
mentale que par leur statut de contre-exemples ou de bouc-émissaires dans la logique régulatoire moderne. Ce construit social, qui ferait du criminel, du fou, de l'handicapé, voire du pauvre, une sorte d'Autre de la société des bien-pensants ou des citoyens «intégrés», ne constitue pas en effet une unité catégorielle qui entretiendrait un quelconque rapport d'extériorité avec le reste de la société. Plus précisément, il faudrait se demander si l'exclusion, comme effet secondaire des choix fondamentaux d'une société foncièrement inégalitaire, est un processus créateur d'altérité. Il s'agit moins d'accepter ou de refuser un "Autre» qui s’imposerait à la société comme de l'extérieur, que de gérer des catégories sociales conçues (et souvent voulues) comme profondément dépendantes. L'image spatiale de l'exclusion, la métaphore tout aussi spatiale de la césure ainsi créée au sein de la société ${ }^{1}$ ne sont justement que cela, des métaphores masquant plus qu'éclairant le processus à l'œuvre ici. Le "peuple» des pauvres, des aliénés et des criminels, même stigmatisé en catégorie sociale spécifique, constitue bien plus qu’une figure de l'altérité: il est davantage la marque d'un manque que le signe d'une intrusion du mal; il illustre avant tout un glissement aux marges de la société "normale», et non, n'en déplaise à certaines élites hystériques de l'époque ${ }^{2}$, un assaut frontal du barbare et de l'inconnu. En ce sens, marginalité n'est pas extériorité, encore moins altérité, mais tout au plus excentricité. Il n'est donc pas sûr que l'usage du concept d'altérité dans ce domaine nous aide beaucoup.

\section{LE PASSÉ COMME «AUTRE»}

Il nous semble pertinent de réfléchir sur le caractère heuristique du concept d'altérité sous un angle tout à fait différent, qui touche un autre aspect, autrement fondamental, de notre pratique historienne. Il s'agit d'examiner l'hypothèse d'une altérité radicale de l'histoire elle-même, comme réalité passée et processus de changement.

1. On pense à l'image forte des "deux nations», celle des pauvres et celle des riches, divisant l'Angleterre industrielle du milieu du xix ${ }^{\mathrm{e}}$ siècle, image tracée par Gladstone dans Sybil, or the Two Nations (Oxford, Oxford University Press, 1845), 1926.

2. Cette crainte profonde de la menace sociale représentée par le "paupérisme» des masses se manifeste en effet parfois avec une sèche brutalité. Ainsi, on retrouve dans le célèbre Dictionnaire de l'économie politique de Coquelin et Guillaumin, en 1853, un article sur la responsabilité des pauvres, article intitulé de façon révélatrice "Parasites». Voir M. Renouard, "Parasites», dans Charles Coquelin et G. V. Guillaumin dir., Dictionnaire de l'économie politique (Paris, Guillaumin, [1853]), 2: 323-329. 
Le prétexte en est donné par la lecture d'un ouvrage fascinant, l'étude de Bartolomé Clavero sur le don et l'échange à l'époque moderne ${ }^{3}$. Dans ce travail, Clavero insiste sur l'irrémédiable étrangeté du monde dans lequel le don et l'échange (notamment dans sa forme extrême de l'usure) sont pensés, aux $\mathrm{xvI}^{\mathrm{e}}$ et $\mathrm{XvII}^{\mathrm{e}}$ siècles supposément source de notre "modernité». Mais ce qui importe ici est moins la démonstration de Clavero que la conclusion épistémologique qu'il en tire:

Je prétendais pénétrer la société d'alors et, finalement, il se trouve que nous n'y avons aucun accès [...]. La recherche qui veut connaitre un autre temps est incapable d'échapper au sien propre. Son objectif secret et son engagement explicite ont été de lutter contre les effets de miroir et d'éviter que l'histoire ne se réduise à la reconnaissance du même, se rendant ainsi incapable de connaitre l'autre. Mais il se trouve que la traversée du miroir n’a servi qu'à se retrouver soi-même. Cette déroute est menée à bon port et cet échec recèle un succès. L'image de soi cesse d'être aplatie, elle a pris du relief. Entre les deux extrêmes que sont la connaissance de l'autre et la reconnaissance de soi, il existe un moyen terme, finalement plus important, c'est-à-dire la connaissance du même, la connaissance de soi. C'est ma dernière illusion ${ }^{4}$.

Et en fin de volume, l'auteur conclut:

L'histoire nous est étrangère et la science historique est à nous. Ce n'est pas celle-là que celle-ci donne à connaître. Et le problème ne tient pas seulement à la question de la connaissance. L'histoire nous situe dans un monde autre que nous nous efforçons de récupérer. Nous nous caractérisons par le fait que nous traitons des étrangers comme des semblables, sous prétexte qu'ils sont nos ancêtres. Notre prochain actuel, quant à lui, s'il appartient à une autre culture, nous demeure étranger 5 .

On pourra bien, comme il est trop souvent de mode dans la pratique de notre métier, reléguer une telle position au rang des élucubrations postmodernistes. Mais on aura tort, et cela pour deux raisons.

D’abord, l'ouvrage, préfacé par Jacques Le Goff, historien peu suspect du péché de postmodernisme et qui parle pourtant de "grand livre ${ }^{6}$ », a

3. Bartolomé Clavero, La grâce du don. Anthropologie catholique de l'économie moderne (Paris, A. Michel, 1996).

4. Ibid., 2.

5. Ibid., 195. Nos italiques.

6. Bien sûr, Le Goff émet de sérieuses réserves face au caractère radical des positions de Clavero: «L'anthropologie qui serait l'objet de la science sociale fondamentale est-elle une théologie dont la référence ne serait plus Dieu mais l'homme? [...] L'histoire "objective" existe-t-elle en dehors de cette histoire-historiographie dont l'impuissance à connaitre le passé se veut ici démontrée? Doit-elle renoncer à établir de façon critique, comme le voulait Marc Bloch, des 
très peu à voir avec cette approche. Il s'agit d'une étude patiente et rigoureuse des textes religieux et juridiques touchant l'usure à l'époque moderne, ne remettant jamais en cause la «réalité » du discours à l'étude et encore moins celle des relations sociales que sous-tend ce discours. Plus encore, Clavero ne doute jamais de la pertinence empirique de l'interprétation qu'il avance. Loin de rejeter la possibilité d'une étude systématique du passé, Clavero propose plutôt un renouvellement de nos pratiques analytiques en promouvant une «anthropologie» des périodes passées.

Mais il y a une deuxième raison, autrement importante, pour laquelle on aurait tort de rejeter comme absurde la position de Clavero. C'est que cette position illustre de façon remarquable, à mon sens, la crise de l'interprétation historique qui traverse notre modernité et, par là-même, la question de notre rapport au passé.

On a souvent fait remarquer que l'histoire est la seule discipline où le même terme d' «histoire» sert à signifier à la fois l'objet sous étude et la pratique disciplinaire qui s'attache à cet objet. Ce phénomène n'est pas fortuit. Il révèle au contraire combien la pratique historienne se distingue bien moins par le type de méthode d'investigation qu'elle applique à l'objet que par le fait qu'elle revendique le savoir total qui s'attache à une dimension existentielle, soit le passé dans son ensemble. Dit autrement, l'historien et l'historienne ne font pas reposer leur légitimité disciplinaire sur des procédés analytiques spécifiques à l'étude d'un segment particulier de l'activité humaine, comme le fait chaque science sociale et humaine: ils prétendent plutôt connaître et analyser tout ce qu'a d'humain le passé, tout ce que la diachronie, comme forme d'existence, a à nous apprendre.

Mission évidemment impossible, non pas tant par le caractère inachevé et incomplet de nos méthodes que par l'immensité de l'objet visé. Cet objet impossible de l'histoire donne lieu à deux réactions diamétralement opposées: 1. Le passé est déclaré impensable comme totalité. Deux raisons peuvent être avancées pour affirmer cette impossibilité de penser le passé dans sa totalité. D’abord celle qui tient que cette totalité passée n'existe tout simplement pas. C'est évidemment la position par excellence de l'approche postmoderniste: le passé n'est que bruit et fureur ou, pour reprendre les termes d'Alain Robbe-Grillet quand il parle du réel: «Le réel

relations réciproques entre le passé et le présent? Les historiens doivent-ils renoncer aux méthodes et aux outils qui ont été créés après les époques qu'ils étudient? Ne pouvons-nous échapper à une incapacité à reconnaître les hommes du passé comme autres? Sommes-nous condamnés à l'anachronisme et à des prisons qui ne sont plus les prisons braudéliennes de la longue durée, mais les prisons du présent, du contemporain? [...] Ne peut-on refonder l'histoire qu'en l'immobilisant? ", Jacques Le Goff, préface à Bartolomé Clavero, ibid., xvi-xvii. 
est discontinu, formé d'éléments juxtaposés sans raison dont chacun est unique, d'autant plus difficiles à saisir qu'ils surgissent de façon sans cesse imprévue, hors de propos, aléatoires ${ }^{7}$.»

Tout au plus peut-on prendre les événements du passé pour ce qu'ils sont, et reconstruire les bribes d'empirie qui se donnent comme faits historiques plus ou moins éclatés, en arrachant ainsi au passé ces morceaux de positivité (et de bravoure!) que sont les études de cas historiques, sans chercher à aller plus loin.

En direct corollaire, le passé est vu comme impensable, en tant que totalité, pour une deuxième raison, qui se fonde, en la contestant, sur la tradition herméneutique, et proclame l'irréductible distance qui nous sépare du passé, distance par définition infranchissable sans la médiation du langage et de l'effort historien d'interprétation du réel. Combien ont souligné la vanité de l'objurgation rankienne d'une histoire qui se doit de dire ce qui s'est véritablement passé ("wie es eigentlich gewesen») ? Devant cette hypothèque incontournable du temps, la réaction de certains sera de vouloir prendre le passé comme à ses propres mots, et s'en tenir le plus possible au sens que le passé donne de lui-même. En somme, il s'agit pour l'historien de s'oublier le plus possible pour en arriver à une écoute épurée, quitte à faire inlassablement la chasse aux «anachronismes ${ }^{8}$ ».

Il n'y a donc, malgré ce qu'on affirme trop souvent, nulle opposition nécessaire entre la négation postmoderne d'une totalité accessible à l'esprit, et le repli positiviste sur le factuel constaté, au nom du «respect» de l’intégrité du passé.

7. Alain Robbe-Grillet, cité dans Pierre Bourdieu, "L'illusion biographique», Actes de la recherche en sciences sociales, 62-63 (juin 1986): 70. On peut aussi citer la formulation frappante d'Harlan: "History will always be what it always has been: the unrecorded and unredordable hum of discontinuous coïncidence", David Harlan, The Degradation of American History (Paris, University of Chicago Press, 1997), 41. Voir aussi Keith Jenkins, Why History? Ethics and Postmodernity (Londres, New York, Routledge, 1999).

8. C'est, de façon un peu contradictoire, la position de Clavero: «Nous ne savons rien et ne devons rien tenir pour connu si nous abordons un domaine réellement étranger [...]. C'est l'objet de l'observation et non le sujet enquêteur qui livrera alors des enseignements. Et ce principe de méthode détermine déjà la substance de l'objet. C'est l'ignorance qui implique l'anthropologie: nous ignorons quelles étaient les notions fondamentales de leur culture. Nous ne savons rien, absolument rien, de la formule anthropologique qui caractérise cette société humaine ancienne: l'ingénuité n'est pas feinte ni la perplexité affectée», B. Clavero, op. cit., 42. Ainsi, l'anthropologie de Clavero serait fondamentalement positiviste dans sa méthode (l'«observation») et nihiliste dans sa visée totalisante (le passé irrémédiablement inconnu)! Sur cette version extrême de la positivité historique dans l'historiographie québécoise, voir la prise de position de mon collègue Donald Fyson et de moi-même : «De la manipulation à l'abdication historienne», Le Devoir, 10 juin 1999. 
2. La position adverse revendique la possibilité d'une appréhension "réelle» du passé tel qu'en lui-même. Ici, les instruments aux mains des historiens sont des instruments d'un savoir vrai, ou du moins véridique, un savoir qui dépasse la simple constatation des choses passées pour en arriver, ne serait-ce que tendanciellement, à un sens général, sinon global. On retrouve ici l'appel célèbre d'un Lucien Febvre à une histoire globale, une histoire qui, dans l'entrelacs des mille questions qu'on lui pose et grâce à la convocation du savoir des autres sciences humaines, permet d'espérer, ne serait-ce seulement que comme objectif à atteindre, un savoir global de la substance du passé. Savoir cumulatif et tendanciel, certes, mais savoir dont l'espérance sous-tend toute la discipline historique depuis trois quarts de siècle au moins. Ici, l'empirisme des historiens est au service d'une mission, et non pas la forme résiduaire d'une pratique désabusée, en deuil d'une totalisation impossible. Cette vision a comme instruments de guerre une méthode revendiquée, et comme morale une rigueur analytique proclamée. Ici, l'histoire est processus de production de sens et totalisation, comme le verra bien Lévinas:

La totalisation ne s'accomplit que dans l'histoire - dans l'histoire des historiographes - c'est-à-dire chez les survivants. Elle repose sur l'affirmation et sur la conviction que l'ordre chronologique de l'histoire des historiens, dessine la trame de l'être en soi, analogue à la nature. Le temps de l'histoire universelle demeure comme le fond ontologique où les existences particulières se perdent, se comptent et où se résument, au moins, leurs essences 9 .

Entre l'impossible synthèse et la potentielle totalisation, entre un savoir qui ne peut être qu'éclaté et la marche conquérante de l'histoire globale, entre la renonciation à penser l'histoire dans sa cohérence et les illusions du savoir cumulatif, existe-t-il une voie médiane, un rapport au passé qui

9. Emmanuel Lévinas, Totalité et infini. Essai sur l'extériorité (Paris, Librairie générale française, (1971) 2001), 48. Il est à noter que chez Lévinas, l'histoire se réduit généralement à ce processus de connaissance et de totalisation : l'histoire est ici à la fois passé et passé reconstruit par les «historiographes" à la recherche d'une impossible totalisation. Mais il peut aussi exister une autre forme de la diachronie: "Cette impossibilité de la totalisation n’est pas purement négative. Elle dessine une relation nouvelle, un temps diachronique qu'aucune historiographie ne transforme en simultanéité totalisée et thématisée et dont l'accomplissement concret serait la relation d'homme à homme, la proximité humaine, la paix entre les hommes, telle qu'aucune synthèse se produisant au-dessus de leurs têtes ou derrière leur dos ne saurait dominer, relation qui, dans les formes où elle semble se produire sous les espèces d'un État, puise encore son sens dans la proximité humaine. L'humanité ne serait pas, dans cette conception, un domaine d'entre les domaines du réel, mais la modalité sous laquelle la rationalité et sa paix s'articulent tout autrement que dans la totalité», Emmanuel Lévinas, Totalité et totalisation (1995), http://www.philagora. net/philo-fac/levinas.htm. 
puisse à la fois témoigner de son irréductible absence et de sa présence têtue?

Tout se passe comme si l'histoire, comme présence-absence du révolu, était l'Autre de notre quête de sens. L'histoire, la substance du passé, est "Autre» en ce sens que la coexistence simultanée des êtres et des choses dans le temps institue une logique sociale qui donne sens à cette concomitance, un sens que l'on peut approcher, que notre esprit peut appréhender, dont notre savoir peut donner une idée, mais sans jamais que nous puissions en avoir la connaissance spontanée et immédiate. Ce qui est "Autre» ici n'est pas tant la différence empirique des êtres et des choses, que la logique globale qui les institue comme société historique donnée. Comme nous ne sommes plus en mesure de «vivre» cette logique désormais révolue, on ne peut que l'approcher en esprit, dans un effort de pensée qui écarte toute paresse analytique, comme tout renoncement dépité à penser autrement les êtres du passé: un effort de pensée conscient, en même temps, des importantes limites de son efficience, irrémédiablement voué au recommencement et à l'incertitude.

Quand un des grands penseurs de l'altérité comme Lévinas parle de $l^{\prime}$ 'Autre», il parle de transcendance, de recherche d'infini ${ }^{10}$, de rapport avec ce qui est radicalement et irrémédiablement étranger à soi. Cet Autre peut se dire comme le non-dit de toute action, comme conscience aiguë de notre non-solitude au monde. Solidarité nécessaire, plus que jamais, avec tous ceux qui ne sont pas moi, mais qui partagent mon présent. Mais solidarité qui n'est pas vraiment un partage avec autrui, qui reste, lui, une présence extérieure qui nous domine et à laquelle on réagit sans pouvoir jamais l'atteindre. Citons encore Lévinas: «La relation avec l'autre n'est pas une idyllique et harmonieuse relation de communion, ni une sympathie par laquelle nous mettant à sa place, nous le reconnaissons comme semblable à nous, mais extérieur à nous; la relation avec l'autre est une relation avec un Mystère ${ }^{11}$.»

10. «Entre une philosophie de la transcendance qui situe ailleurs la vraie vie à laquelle l'homme accèderait, en s'échappant d'ici, aux instants privilégiés de l'élévation liturgique, mystique ou en mourant - et une philosophie de l'immanence où l'on se saisirait véritablement de l'être quand tout «autre» (cause de guerre) englobé par le Même, s'évanouirait au terme de l'histoire, nous nous proposons de décrire, dans le déroulement de l'existence terrestre, de l'existence économique comme nous l'appelons, une relation avec l'Autre, qui n'aboutit pas à une totalité divine ou humaine, une relation que n'est pas une totalisation de l'histoire, mais l'idée de l'infini. ", Emmanuel Lévinas, Totalité et infini... (Paris, Librairie générale française, (1971) 2001), 44.

11. Emmanuel Lévinas, Le temps et l'autre (Paris, Presses universitaires de France (1948) 1983), 63. On retrouve aussi cette notion chez Kierkegaard: «Affirmer l'Autre absolu, comme l'enseigne Kierkegaard, revient bien à affirmer l'existence et la vérité de l'existence. L'absurdité de celle-ci 
En extrapolant à partir de la pensée de Lévinas sur le temps ${ }^{12}$, sans nécessairement y adhérer, il est possible de voir dans le passé une autre dimension de cette radicale altérité qu'il nous décrit. Pourquoi la conception de l'altérité, en effet, devrait-elle se contenter de la métaphore spatiale de l'extériorité, du face à face impliqué par la présence, en synchronie, de l'Autre? L'immédiateté de la confrontation à l'autre constitue-t-elle la seule forme de l'étrange, voire de l'aliénation pour l'esprit humain? En effet, l'Autre, ce n'est pas seulement une forme de la présence du monde autour de nous. C'est aussi ce qui nous affronte et nous interpelle à travers le temps. Lévinas lui-même insiste sur la prise en compte du temps dans la conception de l'Autre, notamment quand il réfléchit au rapport entre l'avenir et l'altérité: "Quand on enlève au présent toute anticipation, l'avenir perd toute conaturalité avec le présent [...] Il est absolument autre et nouveau. Et c'est ainsi qu'on peut comprendre la réalité même du temps, l'absolue impossibilité de trouver dans le présent l'équivalent de l'avenir, le manque de toute prise sur l'avenir ${ }^{13}$.»

En ce sens, le passage du temps est facteur d'altérité : il ouvre une porte sur l'étranger, l'inconnu : altérité issue du changement incontournable dans l'ordre des choses et des êtres. Mais l'avenir n'est «Autre», dans ce cas de figure, que parce qu'il n'est pas encore advenu. Son étrangeté fondamentale ne vient pas tant du temps qui nous sépare de lui que du fait qu'il est "ouvert» à mille réalités et possibles éventualités. L'Autre de l'avenir est en ce sens non seulement impensable, mais inabordable parce qu'il est indéterminé et virtuel.

ne se résout nullement à l'aune d'une identité conservée, simplement redéployée par le savoir, comme le voulait Hegel; au contraire c'est le surgissement imprévisible de l'Autre qui donne sens à l'existence, au-delà de tout savoir, même si cette altérité est dans un premier temps refusée, puis acceptée sous la figure d'un Autre absolument Autre», Didier Moulinier, Existence et altérité, http://perso.club-internet.fr/tuyau/Etudes/critiques_du_sujet/sujet-existence.htm

12. On l'a vu, Lévinas assimile en général l'histoire à la constitution toujours fragile d'une totalité par les historiens, donc comme un travail de pensée, qu'il oppose à la quête de l'infini et de transcendance impliquée par notre rapport à l’Autre. Autrement, le passé «pour soi » se réduit à l'infinité du réel et des faits: «L'historique est à jamais absent de sa présence même. Nous voulons dire par là qu'il disparaît derrière ses manifestations - son apparition est toujours superficielle et équivoque, son origine, son principe, toujours ailleurs. Il est phénomène - réalité sans réalité [...] La connaissance du thématisé n'est qu'une lutte recommençante contre la mystification toujours possible du fait; à la fois, une idolâtrie du fait, c'est-à-dire une invocation de ce qui ne parle pas, et une pluralité insurmontable de significations et de mystifications», Emmanuel Lévinas, Totalité et infini... (Paris, Librairie générale française, (1971) 2001), 60.

13. Emmanuel Lévinas, Le temps et l'autre (Paris, Presses universitaire de France, (1948) 1983), 71. Il déclare aussi, encore plus lapidairement: «L'avenir, c'est l'Autre», Ibid., 64. 
L'Autre impliqué par le passé est par contre tout différent, en ce qu'il représente une tout autre manifestation du temps. Le temps ne s'ouvre pas ici sur des possibles inconnus, mais se renferme dans les limites de l'advenu, du révolu. Cette conscience du révolu et du rapport problématique qu'il entretient avec notre présent est encore exacerbée par les temps démocratiques qui sont avec nous. On pourrait dire, en allant vite, que la démocratie a inventé l'histoire comme rapport à l'altérité.

Et ce, d'abord parce que la démocratie est un mode de régulation humaine qui génère, par définition, l'incertitude du temps, qu'il soit vécu, mémorisé ou appréhendé. Claude Lefort s'est exprimé éloquemment làdessus: «L'essentiel, à mes yeux, est que la démocratie s'institue et se maintient dans la dissolution des repères de la certitude. Elle inaugure une histoire dans laquelle les hommes font l'épreuve d'une indétermination dernière, quant au fondement du Pouvoir, de la Loi, et du Savoir, et au fondement de la relation de l'un avec l'autre, sur tous les registres de la vie sociale ${ }^{14}$.»

Mais cette indétermination dont nous parle Lefort est aussi temporelle, dans la mesure où ce sont les rapports avec le passé et l'avenir qui deviennent fondamentalement indéterminés. Les temps anciens avaient inventé un passé à la mesure d'une diachronie prédéterminée par la foi : temps de la prédestination vers la rédemption à venir ou bien temps répétitif de la tradition à honorer et à reproduire. Dans cette logique temporelle, l'histoire pouvait difficilement être autre chose que la confirmation circonstancielle de la morale et de la puissance de l'au-delà, ou un réservoir d' "exempla ", de modèles à suivre (ou à éviter) au service de la vie présente meilleure et en attendant une vie future transcendant l'existence terrestre. Pas de place dans cette vision pour l'étrangeté de l'altérité temporelle, pour un monde passé et à venir marqué par la différence fondamentale avec le présent.

Les temps démocratiques ouvrent, par le biais de l'indétermination impliquée par le règne sans partage de la liberté, un espace rendant possible la perte de repères, forme suprême de la solitude. Tocqueville a été sensible à cette dimension nouvelle de la vie en société libérale: «Ainsi, non seulement la démocratie fait oublier à chaque homme ses aïeux, mais elle lui cache ses descendants et le sépare de ses contemporains; elle le

14. Claude Lefort, "La question de la démocratie», Essais sur le politique $\mathrm{XIX}^{\mathrm{e}}-\mathrm{XX}{ }^{\mathrm{e}}$ siècles (Paris, Seuil, 1986), 29. C’est Lefort qui souligne. 
ramène sans cesse vers lui seul et menace de le renfermer enfin tout entier dans la solitude de son propre cœur ${ }^{15}$.»

«Oublier ses aïeux», dans ce sens, c'est perdre la certitude que le passé est garant de l'avenir, douter qu'il constitue vraiment un réservoir de sagesse apte à guider le présent. Nouvelle expérience, traumatisante, de l'histoire devenue étrangère que l'historien Reinhart Koselleck a bien rendue : «La découverte rétroactive de l'altérité absolue du passé constitue l'expérience singulière de notre propre histoire dans son élaboration herméneutique ou socio-scientifique ${ }^{16}$."

D’où l'enjeu fondamental représenté par la (re) découverte du passé par une pratique historienne renouvelée. Il s'agissait de retrouver le fil diachronique qui soit en mesure d'expliquer notre présent, la pleine conscience de ce présent permettant d'éclairer l'histoire de sa lumière rétrospective. On rêvait de reconstruire la trame subtile qui permettait de faire émerger des temps tyranniques les premières lueurs de la liberté. De refaire le parcours où se dessinait, déjà, la trajectoire de la nation. De faire sens de ces siècles de noirceur et d'oppression à la lumière de l'actuel. Enjeu essentiel, qui permettait de faire sens de l'histoire en maîtrisant son altérité fondamentale, ou plutôt en masquant cette altérité derrière la familiarité combien rassurante induite par la connaissance produite de ce passé. Il s’agissait bien, comme le dirait Lévinas, de posséder ce passé, de réduire son impossible diversité en le thématisant et en le totalisant.

Dans des lignes fascinantes, Koselleck a décrit ce nouveau rapport à la temporalité né de l'époque des Lumières, soit une conception du temps qui faisait du présent le moment où s'actualisaient tout à la fois un espace d'expérience, regard rétrospectif sur le passé, et un horizon d'attente, aspiration à devenir qui réduisait l'indétermination du futur ${ }^{17}$. Cette histoire «au service» du présent «éclairait» à son tour ce présent en nous inscrivant dans une diachronie précise qui nous permettait d'espérer maîtriser, dans la mesure du possible, le cours du temps...

15. Alexis de Tocqueville, De la démocratie en Amérique (Paris, Garnier-Flammarion, (1840) 1981), 1: 127. À rapprocher de cette affirmation de Lévinas: «La solitude est une absence de temps», Emmanuel Lévinas, Le temps et l'autre (Paris, Presses universitaires de France, (1948) 1983), 38. Sur ce sujet, voir Jean-Marie Fecteau, «Sur les conditions historiques de production de la solitude moderne ", Revue internationale d'action communautaire, 29 (printemps 1993): 17-21.

16. Reinhart Koselleck, L'expérience de l'histoire (Paris, Gallimard/ Seuil/EHESS, 1997), 212 213.

17. Reinhart Koselleck, Le futur passé. Contribution à la sémantique des temps historiques (Paris, Éditions de l'École des hautes études en sciences sociales, 1990). 
Cependant tout cela s'accomplissait au prix d'une familiarité abusive, d'une réduction plus ou moins arbitraire de l'étrangeté fondamentale du "monde que nous avons perdu », pour reprendre le titre du célèbre livre de Peter Laslett ${ }^{18}$. Finalement, est-il nécessaire de réduire l'histoire à ce que nous en savons empiriquement et à ce qu'il est possible d'en savoir? Peut-il exister un autre rapport à cette histoire que la simple connaissance, même enrobée d'empathie? Peut-on tracer les contours de l'inconnu, les modalités de l'étrangeté, sonder la profondeur de l'Autre historique, ne serait-ce que pour mieux mettre en relief le présent, mieux le confronter dans sa nouveauté profonde par rapport à ce qui a été? Découvrir des logiques autres, non pas aux confins du monde mais aux limites du temps, non pas pour les imiter et les prendre pour exemples, mais pour les contraster à notre logique propre, permettant par là-même une mise en relief de cette logique du présent?

Est-il possible aussi de retrouver, par l'étude du processus de mise en place de ces logiques sociales étrangères et par l'analyse de leur dissolution, non pas l'«explication» du changement qui nous donnerait comme l'impossible clef de l'avenir, mais ne serait-ce que la dynamique propre qu'impulse le changement social?

Quel type de savoir pourra-t-on retirer de cette quête? Ici encore, l'avenir nous confronte dans son étrangeté fondamentale. Mais oser confronter l'histoire des hommes et des femmes comme altérité radicale importe pour la suite du monde. C'est peut-être la condition d'une nouvelle humilité face à l'histoire, comme de la nécessité de la penser autrement. Ce qui nous ramène, paradoxalement peut-être, à Foucault, et à ces paroles qu'il adressait, l'année de sa mort, il y a 20 ans, à ses nombreux critiques (et où je me suis permis - on m'excusera ce manque de rigueur... - de remplacer la référence à la philosophie par celle à l'histoire):

Quant au motif qui m’a poussé [...] c'est la curiosité, - la seule espèce de curiosité, en tout cas, qui vaille la peine d'être pratiquée avec un peu d'obstination: non pas celle qui cherche à s'assimiler ce qu'il convient de connaître, mais celle qui permet de se déprendre de soi-même. Que vaudrait l'acharnement du savoir s'il ne devait assurer que l'acquisition des connaissances, et non pas, d'une certaine façon et autant que faire se peut, l'égarement de celui qui connaît? Il y a des moments dans la vie où la question de savoir si on peut penser autrement qu'on ne pense et percevoir autrement qu'on ne voit est indispensable pour continuer à regarder ou à réfléchir. On me dira peut-être 
que ces jeux avec soi-même n'ont qu'à rester en coulisses; et qu'ils font, au mieux, partie de ces travaux de préparation qui s'effacent d'eux-mêmes lorsqu'ils ont pris leurs effets. Mais qu'est-ce dont que [l'histoire] aujourd'hui - je veux dire l'activité [historique] - si elle n'est pas le travail critique de la pensée sur elle-même? Et si elle ne consiste pas, au lieu de légitimer ce qu'on sait déjà, à entreprendre de savoir comment et jusqu'où il serait possible de penser autrement? Il y a toujours quelque chose de dérisoire dans le discours [historique] lorsqu'il veut, de l'extérieur, faire la loi aux autres, leur dire où est leur vérité, et comment la trouver, ou lorsqu'il se fait fort d'instruire leur procès en positivité naïve; mais c'est son droit d'explorer ce qui, dans sa propre pensée, peut être changé par l'exercice qu'il fait d'un savoir qui lui est étranger ${ }^{19}$.

\section{CONCLUSION}

Il faudrait, pour combler, de façon toujours incomplète, ce manque qu'est notre rapport au passé, savoir inventer, par retouches délicates, comme des reconstitutions imaginées tenant à la fois des certitudes empiriques, même temporaires et localisées, et des rapports sociaux conçus par l'opération de la pensée. Il faudrait que l'histoire, comme discipline, investisse un territoire, à la fois malaisé et mouvant, entre la description et l'invention, empruntant, dans leur différence, tant au roman qu'à l'analyse du réel, s'acceptant ainsi, comme le promouvait naguère Ricoeur ${ }^{20}$, comme un récit vrai et en même temps inventé.

Car s'il peut y avoir "échange», il ne peut y avoir dialogue avec le passé. L' «Autre» du vivre immédiat est un existant qui nous interpelle dans un appel qui trace, comme en négatif, les contours de notre identité, de notre

19. Michel Foucault, Histoire de la sexualité, II : L'usage des plaisirs (Paris, Gallimard, 1984), 15-16. On pourra confronter cette exigence avec le pessimisme un peu désespéré de Clavero : "Ce qu'il faut, ce n'est pas une histoire réintégrée avec toutes ses dépendances mais une histoire intégrale avec toute son autonomie. Ou alors, l'histoire, le passé de notre présent, n'est pas ce dont nous avons besoin. La science historique est née comme une morale pas exactement favorable à la liberté [...]. Pour ce qui nous intéresse, le problème dépasse celui de la défiguration du passé pour toucher les dysfonctionnements du présent: un droit de la liberté appelle une morale de l'individu, autrement dit du genre, une morale qui puisse être enseignée, non pas en fonction d'une culture du particulier mais en fonction d'un impératif de vie en commun, virtuellement universelle. C'est de cela que nous avons besoin. Ce n'est pas l'histoire, qu'elle se veuille science ou qu'elle dissimule sa nature morale, qu'elle soit cultivée comme une profession ou vénalisée comme un passe-temps, qui pourra nous l'apporter. La question est strictement et tristement contemporaine. Les semblables sont les contemporains pour éloignées que soient les différentes cultures. L’anthropologie qui doit nous intéresser est celle qui peut être commune. Les étrangers doivent être nos ancêtres, avec les legs de leur culture, socialement précieuse pour leur époque mais désormais inutilisable pour la nôtre, dans la recherche de la vie en commun.», B. Clavero, op. cit., 196-197.

20. Paul Ricoeur, Temps et récit (Paris, Seuil, 1983-1985), 3 vol. 
définition de soi (individuel comme collectif). Cet «Autre» est celui dont la réalité se déplace au rythme de notre rapport à lui. Il est celui qui nous parle et nous provoque, et même notre rejet de lui est une relation au défi qu'il représente.

L'“Autre» du passé, par contre, est comme le discours à la fois cacophonique et définitif du révolu, un discours mort en ce que ceux et celles à qui il s'adressait dont aussi irrémédiablement repoussés dans le déjàarrivé. L'autre du passé est comme la somme des identités révolues qui lentement s'estompent dans la mémoire courte des générations. Cet "Autre» temporel ne constitue nullement, comme l' «Autre» de la synchronicité, cette différence contrariante qui, confrontée à nous, nous permet de s'adresser à elle en tant que différence tout en nous construisant dans cette confrontation. L' «Autre» du passé est la différence radicale, irrémédiable, celle qu'on ne peut saisir que par cette mixture de connaissance empirique et d'empathie rétrospective unique à la pratique historienne (et qui la distingue par là-même de la mémoire, même collective).

Le rapport à cet autre radical du passé révolu ne peut donc être que celui de la reconstruction à la fois nécessaire et impossible d'un réel disparu dans les méandres du temps. La tâche de l'historien et de l'historienne face à cet "Autre» est donc à la fois un exercice de lucidité et d'imagination. Un composé explosif qui génère un sens qui seul nous permet de nous repérer - et de nous démarquer - dans le temps des humains.

Car il n'est nullement vrai que l'oubli (ou son triste corollaire savant: le refus de confronter le passé comme objet de savoir et instance du réel) est la réponse pertinente à l'appel du futur de nos temps postmodernes. À un passé négligé répond toujours un avenir sans aspirations. Passé et avenir, héritage et destin, ces deux «autres» de la temporalité humaine, ne peuvent devenir vivants que s'ils sont confrontés à notre désir de comprendre, lié à notre faculté d'espérer. 